\title{
Bertrand Equilibria and Sharing Rules
}

\author{
Steffen H. Hoernig* \\ School of Economics, Universidade Nova de Lisboa \\ Campus de Campolide, 1099-032 Lisboa, Portugal \\ CEPR, London
}

March 2005

JEL: C72, D43, L13

*shoernig@fe.unl.pt, tel. +351-213801645, fax +351-213870933. I would like to thank Fernando Branco for helpful discussions, the audience at Pompeu Fabra (Barcelona), ISEG (Lisbon), University of Mannheim, ESEM 2003 (Venice), two anonymous referees, and the editor Dan Kovenock for useful comments. This research received financial support under project POCTI/ECO/37925/2001 of FCT and FEDER. 


\begin{abstract}
We analyze how sharing rules affect Nash equilibria in Bertrand games, where the sharing of profits at ties is a decisive assumption. Necessary conditions for either positive or zero equilibrium profits are derived. Zero profit equilibria are shown to exist under weak conditions if the sharing rule is "sign-preserving". For Bertrand markets we define the class of "expectation sharing rules", where profits at ties are derived from some distribution of quantities. In this class the winner-take-all sharing rule is the only one that is always sign-preserving, while for each pair of demand and cost functions there may be many others.
\end{abstract}

JEL: C72, D43, L13

Keywords: Bertrand games, Sharing rule, Tie-breaking rule, Sign-preserving sharing rules, Expectation sharing rules 


\section{Introduction}

Existence and multiplicity of pure and mixed strategy Nash equilibria in Bertrand pricing games have been widely researched. The results obtained depend on properties of the demand and production cost specifications, and on the sharing or tie-breaking rule embodied in the payoffs. While the former have been the subject of some investigation, the latter went almost unnoticed. This is surprising because the sharing rule has a decisive role to play because candidate equilibria will often involve ties at the lowest price.

The contribution of our research is two-fold. First, we consider "Bertrand games" with generic payoffs, not necessarily derived from some demand and cost function. Second, we put the sharing rule into the foreground. No a priori restrictions are imposed on their shape; rather, we discover which properties that have an effect on equilibria.

In doing so, we find necessary conditions on a single player's payoff on the one hand, and on the sharing rules on the other hand, for the emergence of positive or zero equilibrium payoffs for some or all players. The only relevant condition on sharing rules in this context is that they be "tie-decreasing", that is, a tied firm's payoffs should be below a single firm's at the same price. These results are then used to state a general result about the necessity of zero equilibrium profits.

We also demonstrate how to prove quickly whether a given Bertrand game has neither pure nor mixed strategy Nash equilibria. In zero-sum games this is not very difficult: It is enough to prove that the maximin and minmax value differ, see [13]. For non-zero-sum games like Bertrand competition with variable demand, however, this is a surprisingly hard exercise.

We then analyse the class of "sign-preserving" sharing rules. With this 
class of sharing rules, zero-profit Nash equilibria exist if a single firm's payoffs are continuous. Therefore under sign-preserving sharing the classical "Bertrand paradox" of zero profits is restored, with the new interpretation that "price is equal to average cost". The "competitive outcome" of price equal to marginal cost turns out to be the exception rather than the rule, and generically occurs only under constant returns to scale. These results therefore shed new light on the question of "Bertrand paradox" outcomes of zero equilibrium profits, generalizing [8] and [3] to arbitrary payoffs and sharing rules.

Finally, for Bertrand pricing games we define the class of "expectation sharing rules", where a tied firm's payoff is defined as the expectation over some distribution of quantities. We concentrate on the case where the total quantity allocated to firms is equal to quantity demand by consumers. Two known sharing rules are part of this class: The classical sharing rule is "equal sharing", where each firm receives an identical share with certainty. For the case where each firm is equally like to receive all demand, [3] have introduced the notion of "winner-take-all" sharing, but this sharing rule appeared at least in [11] and [2], and is referred to in [14, p. 139]. We show that there are pairs of demand and cost functions for which either all or at least an infinite number of expectation sharing rules are sign-preserving. On the other hand, only the winner-take-all sharing rule is sign-preserving under all circumstances.

The literature on the Bertrand model at first concentrated on constant returns to scale. For two firms and equal sharing, [6] has shown that pricing at marginal cost is the unique equilibrium when demand is bounded, continuous and has a finite choke-off price. With more than two firms there is a continuum of zero-payoff equilibria where at least two firms always choose 
price equal to marginal cost, and the other firms randomize arbitrarily between prices not lower than marginal cost. This multiplicity of equilibria is "inessential" in the sense that equilibrium production, profits and consumer welfare are the same for all these equilibria.

On the other hand, [8] have shown that infinite monopoly payoffs are not only sufficient but also necessary in duopoly for Nash equilibria other than the zero-payoff equilibrium to obtain. In the context of winner-take-all sharing, [2] have also demonstrated the existence of these equilibria, but not shown necessity of infinite monopoly payoffs.

If returns to scale in the Bertrand model are not constant then the situation is much more complicated. [5] showed that under decreasing returns to scale there is a continuum of pure strategy equilibria where firms make positive profits. Later, [7] amplified this result by proving that in this case there are continua of mixed equilibria on finite and continuous supports. On the other hand, below we present an example where under increasing returns to scale neither pure nor mixed strategy Nash equilibria exist.

An alternative approach where sharing rules are part of the equilibrium, rather than part of the definition of the game, is that of "games with endogenous sharing rules" of [12]. Recently, [1] has shown that endogenous sharing rules can be constructed from continuous (instead of discrete) approximations to payoffs, which paves the way to finding them analytically. In this context a sharing rule is some selection from the convexification of payoffs at discontinuities. While there is always a selection such that an equilibrium exists, nothing can be said a priori about the shape of this selection, which may be highly sensitive to the specification of the model.

In Section 2 we set out the model and define sharing rules. Nash equilibria are analysed in Section3. Section4 deals with sign-preserving sharing, while 
Section 5 analyses expectation sharing rules. Section 6 concludes. Almost all proves are in the Appendix.

\section{The Model}

Let $N=\{1, . ., n\}$ be a set of $n$ identical players who simultaneously chooses actions $p \in \mathbb{R}_{+}$. Denote by $T\left(p_{1}, . ., p_{n}\right)=\left\{j \in N \mid p_{j}=\min \left\{p_{1}, . ., p_{n}\right\}\right\}$ the set of "winners", i.e. players tied at the smallest action, and let $\pi_{m}(p)$ denote the payoffs of the $m=\left|T\left(p_{i}, p_{-i}\right)\right| \in N$ winners at action $p$. Players who are not winners receive zero payoffs. With $p_{-i}=\left(p_{1}, . ., p_{i-1}, p_{i+1}, . ., p_{n}\right)$, player $i$ 's payoff is then given by

$$
u\left(p_{i}, p_{-i}\right)=\left\{\begin{array}{ccc}
\pi_{\left|T\left(p_{i}, p_{-i}\right)\right|}\left(p_{i}\right) & \text { if } & i \in T\left(p_{i}, p_{-i}\right) \\
0 & \text { if } & i \notin T\left(p_{i}, p_{-i}\right)
\end{array} .\right.
$$

Let $M=\{2, . ., n\}$. Typically $\pi_{1}$ is defined by the fundamentals of the game, while the $\left\{\pi_{m}\right\}_{m \in M}$ define the sharing rule, incorporating additional assumptions.

The above definition of payoffs can be extended to mixed strategies, that is, cumulative distribution functions, as in [9]. Let $\Delta$ be the set of mixed strategies on $\mathbb{R}_{+}$. If $F_{j} \in \Delta, j \neq i, F_{-i}=\left(F_{1}, . ., F_{i-1}, F_{i+1}, . ., F_{n}\right)$ and $N_{i}=N \backslash\{i\}$, then

$$
u\left(p_{i}, F_{-i}\right)=\sum_{T \subset N_{i}} \pi_{|T|+1}\left(p_{i}\right)\left(\prod_{j \in T} \alpha_{j}\left(p_{i}\right)\right)\left(\prod_{k \in N_{i} \backslash T}\left(1-F_{k}\left(p_{i}\right)\right)\right),
$$

where $\alpha_{j}(p)=F_{j}(p)-\lim _{p^{\prime} \nearrow p} F_{j}\left(p^{\prime}\right)$ is the size of the atom (if any) in $F_{j}$ at $p \in \mathbb{R}_{+}$. The summation is over the sets $T$ of players that may be tied with player $i$ at action $p_{i}$. For any $N^{\prime} \subset N$ and action $p \in \mathbb{R}_{+}$let $A\left(p, N^{\prime}\right)=\left|\left\{j \in N^{\prime} \mid \alpha_{j}(p)>0\right\}\right|$ be the number of players in $N^{\prime}$ who have 
an atom at $p$. The games that we will consider are "Bertrand games" $\Gamma=$ $\left\langle N, \Delta, \pi_{1},\left\{\pi_{m}\right\}_{m \in M}\right\rangle$.

In the Bertrand model players are firms in an industry selling a homogeneous good, and actions are prices. If $D, C: \mathbb{R}_{+} \rightarrow \mathbb{R}_{+}$are a the demand and cost functions, respectively, a single firm's payoffs are $\pi_{1}(p)=$ $p D(p)-C(D(p))$.

More generally, this setup includes all static games of complete information where players are symmetric, strategy spaces are completely ordered, the winners' payoff only depends on the winning action and the number of winners, and any player who plays a higher action receives zero payoffs. This set includes some first-price auctions (after inverting the strategy spaces).

As we defined them, sharing rules are mappings $(p, m) \mapsto \pi_{m}(p)$. For most of the analysis we need not specify this mapping further, but in Section 5 we consider the specific class of expectation sharing rules. By way of comparison, the definition of an endogenous sharing rule of [12] in the specific context of the Bertrand model, roughly, amounts to some arbitrary selection from the convexification of $\pi_{1}$ at its discontinuities. This cannot reproduce the payoffs all Bertrand models with decreasing returns to scale, since payoffs $\pi_{m}$ at ties may lie outside the convex hull of $\pi_{1}$.

Two well-known sharing rules in the Bertrand model are equal sharing and winner-take-all sharing. Equal sharing amounts to $\pi_{m}(p)=p D(p) / m-$ $C(D(p) / m)$ : Each firm receives an equal share of demand. This is the sharing rule traditionally posited. Winner-take-all sharing $W$ implies that one randomly selected firm receives all demand, and results in expected profits $\pi_{m}(p)=\pi_{1}(p) / m$. This latter sharing rule has appeared in [11], [2] and [3]. It is immediate that under constant returns to scale, $C(q)=c q$ for some 
$c \geq 0$, the payoffs under the two sharing rules coincide,

$$
\pi_{m}(p)=p D(p) / m-c D(p) / m=\pi_{1}(p) / m
$$

This equivalence of payoffs under constant returns to scale in the Bertrand model may explain why the subject of sharing rules as such has not yet received much attention. Generally payoffs between these two sharing rules differ, with drastic consequences. If winner-take-all sharing is assumed, existence and uniqueness of symmetric pure strategy equilibria are almost trivial, as shown below. Under equal sharing the situation is much more complicated, with the possible outcomes of non-existence of equilibrium or existence of a continuum of equilibria depending on the returns to scale.

Many reasonable conditions can be imposed on sharing rules. For now we restrict the class of allowable sharing rules as little as possible. Let us first make the following definitions:

Definition 1 A function $f: \mathbb{R} \rightarrow \mathbb{R}$ is

1. zero-at-zero (ZAZ) if $f(x)=0$ if $x=0$;

2. sign-preserving (SP) if $f(x) \gtreqless 0$ if and only if $x \gtreqless 0$ for all $x \in \mathbb{R}$.

A sign-preserving function is necessarily zero-at-zero. Two classes of sharing rules of special interest are:

Definition 2 Given $\pi_{1}$, a sharing rule $\left\{\pi_{m}\right\}_{m \in M}$

1. is sign-preserving if $\pi_{m}(p)$ has the same sign as $\pi_{1}(p)$, for all $m \in M$ and $p \in \mathbb{R}_{+}$;

2. is tie-decreasing at a price $p \in \mathbb{R}_{+}$if $\pi_{1}(p)>\max _{m \in M} \pi_{m}(p)$ whenever $\pi_{1}(p)>0$. It is tie-decreasing if it is tie-decreasing at all $p \in \mathbb{R}_{+}$. 
It follows immediately from this definition that for any strictly increasing and zero-at-zero function $z \in \mathbb{R} \rightarrow \mathbb{R}$ the sharing rule $\left\{z\left(\pi_{m}\right)\right\}_{m \in M}$ is SP if $\left\{\pi_{m}\right\}_{m \in M}$ SP. While equal sharing is not SP if returns to scale are not constant, winner-take-all sharing is $\mathrm{SP}$ for all $\pi_{1}$. Therefore any $z(W)$ is also SP, and there always exist infinitely many SP sharing rules for all payoffs $\pi_{1}$.

A sharing rule is tie-decreasing if payoffs at ties are below a single player's payoff at the same price. The role of tie-decreasing sharing rules emerges when we consider Nash equilibria with positive profits in the next section. Winner-take-all sharing is tie-decreasing, while equal sharing is not under decreasing returns to scale.

\section{$3 \quad$ Nash Equilibria and Sharing Rules}

In this section we will derive general characteristics of Bertrand equilibria, i.e. the Nash equilibria of Bertrand games. We describe whether and how these are related to properties of the sharing rule or of the demand and cost functions. Starting from the assumption that equilibria exist, there is surprisingly much that one can say without presupposing a given sharing rule. We first analyze equilibria involving positive expected profits for some firms, and then state a general theorem that describes when all Bertrand equilibria must involve zero profits.

Let $\left(F_{1}^{*}, . ., F_{n}^{*}\right) \in \mathcal{S}^{n}$ be a Nash equilibrium, with equilibrium payoffs $\Pi_{i}=u_{i}\left(F_{i}^{*}, F_{-i}^{*}\right)$ :

$$
u_{i}\left(F_{i}^{*}, F_{-i}^{*}\right) \geq u_{i}\left(F_{i}, F_{-i}^{*}\right) \forall F_{i} \in S, \forall i \in\{1, . ., n\}
$$

For all $i \in N$ let $S_{i}$ be the support of firm $i$ 's equilibrium strategy $F_{i}$, then $S_{i}$ is closed and bounded from below. Necessarily $u_{i}\left(p_{i}, F_{-i}^{*}\right) \leq \Pi_{i}$ for all 
$p_{i} \in \mathbb{R}_{+}$, and $u_{i}\left(p_{i}, F_{-i}^{*}\right)=\Pi_{i}$ for all $p_{i} \in P_{i}=S_{i} \backslash Z_{i}$, where $Z_{i}$ is a set of $F_{i}^{*}$ measure zero such that $u_{i}\left(p_{i}, F_{-i}^{*}\right)<\Pi_{i}$ for all $p_{i} \in Z_{i}$. The fact there may be prices $p_{i} \in S_{i}$ with $u_{i}\left(p_{i}, F_{-i}^{*}\right)<\Pi_{i}$ complicates some of our arguments below.

We first highlight some of the complexities that can be encountered when the assumption of constant returns to scale is abandoned.

Example: In a market with two symmetric firms demand is $D(p)=$ $\max \{0,1-p\}$, and the cost function is given by $c(0)=0, c(q)=1 / 6$ for $q \in(0,1 / 3]$, and $c(q)=1 / 4$ for $q>1 / 3$. With equal sharing there is no symmetric pure strategy equilibrium, but a pair of asymmetric pure strategy equilibria with $p_{i}=2 / 3$ (at the left-discontinuity) and $p_{j}=1 / 2$, with both firms $i, j$ making zero profits. Furthermore, there is a continuum of mixed strategy equilibria where firm $i$ plays $p_{i}=2 / 3$ with certainty, while firm $j$ randomizes between an atom at $p_{j}=1 / 2$ and prices larger than $2 / 3$. Expected payoffs in this case are $\Pi_{i}=\left(1-\alpha_{2}^{*}(1 / 2)\right) / 18$, and $\Pi_{j}=0$. With winner-take-all sharing, there are two symmetric pure strategy equilibria: $p=1 / 2$ (zero profits), and $p=2 / 3$ (positive profits), and a continuum of asymmetric ones with $p_{i}=1 / 2$ and $p_{j} \in(1 / 2,2 / 3)$ (zero profits).

\section{$<<$ HERE FIGURE $1>>$}

This example shows that one may find equilibria where some firms make positive profits while others make zero profits. This happens even though firms are ex ante symmetric. Furthermore, there may be many such equilibria. We have found equilibria where the firm making zero equilibrium profits sometimes plays lower prices than the firm making positive profits, demonstrating that the "intuition" that only firms charging the lowest price have a chance of making any profit is wrong. 
[5] has shown that there under equal sharing and decreasing returns to scale there may be a continuum of symmetric equilibria involving positive profits. Furthermore, [7] demonstrates that in this case there also are infinitely many mixed equilibria where firms gain positive expected profits and the sets $P_{i}$ are continua. All of this is shown in a context of finite monopoly profits, so that the "bootstrap" results of [2] and [8] do not apply.

Let $J_{>}=\left\{i \in N \mid \Pi_{i}>0\right\}$ and $J_{=}=\left\{i \in N \mid \Pi_{i}=0\right\}$ be the sets of firms with positive and zero expected equilibrium profits, respectively. There can be none with negative profits, given that $C(0)=0$ and deviations to higher prices are always possible. The following lemma lists some of the properties of Bertrand equilibria where some firms have positive profits. These results are independent of the sharing rule. Let "maximum profits" be defined as $\pi^{\max }=\sup _{p \in \mathbb{R}_{+}, k \in N} \pi_{k}(p)$. In the classic Bertrand model with ES and constant marginal costs these are equal to monopoly profits $\sup _{p} \pi_{1}(p)$.

Lemma 1 Given a Bertrand $\Gamma$, assume that $\pi^{\max }<\infty$ and that $\left(F_{1}^{*}, . ., F_{n}^{*}\right)$ is a Nash equilibrium with $J_{>} \neq \emptyset$. Then:

1. There is a price $t \in \mathbb{R}_{+}$such that $t=\max S_{i}$ for all $i \in J_{>}$. If $\left|J_{>}\right|>1$ then $F_{j}^{*}(t)<1$ for all $j \in J_{=}$, and there exists $K \in \mathbb{R}_{+}$ such that $\alpha_{i}^{*}(t) \Pi_{i}=K$ for all $i \in J_{>}$. If $J_{>}=\{k\}$ and $J_{=\neq} \neq \emptyset$, then $F_{j}^{*}(t)<1+\alpha_{j}^{*}(t)$ for all $j \in J_{=}$, and $\alpha_{k}^{*}(t)>0$ or there is a sequence $\left\{p_{l}\right\}_{l=1}^{\infty} \subset S_{k}$ such that $\alpha_{k}^{*}\left(p_{l}\right)>0$ for all $l$ and $\lim _{l \rightarrow \infty} p_{l}=t$.

2. Let $J_{=} \neq \emptyset$. For all firms $i \in J_{>}$the sets $P_{i}$ are countable, and $\sum_{p \in P_{i}} \alpha_{i}^{*}(p)=1$. If for $i \in J_{>}$there is some price $r \in P_{i}$ with $\alpha_{i}^{*}(r)=0$ then it is a limit point of atoms in $P_{i}$, and if $r<t$ then $A^{*}\left(r, J_{=}\right)=\left|J_{=}\right|$. 
3. Let $J_{=} \neq \emptyset$. If $p<t$ with $\pi_{1}(p)>0$ then $A^{*}\left(p, J_{>}\right) \geq 1$ or $A^{*}\left(p, J_{=}\right) \geq$ 2 .

The first result essentially states that the supports of all firms earning positive profits in equilibrium have a joint finite maximum $t$, and that $t$ is an atom for all firms in $J_{>}$if there are at least two of them. zero-payoff firm have have a maximum of their equilibrium support equal to $t$ if $\left|J_{>}\right|=1$, otherwise their support extends beyond $t$ (and may not have a maximum).

The second result imposes a lot of structure on possible equilibria if firms with both positive and zero profits are present. [7] has shown that if all firms make positive profits then the equilibrium $P_{i}$ 's may be uncountable. Lemma 1 explicitly rules this out if there is at least one firm making zero profits, because zero-payoff firms would deviate to these (non-atomic) prices. On the other hand, zero-payoff firms may not want to deviate to prices which already is an atom for some other player: If $m-1$ players play a price $p$ with positive probability then it may still yield positive expected profits to them, but not any more to an $m$ th player.

Some as of yet open questions are: 1 . Can firms in $J_{=}$play atoms where firms in $J_{>}$play atoms? The answer seems to be "yes". 2. Can we say anything about limit points of atoms, in particular $t$ for $\left|J_{>}\right|=1$ ? Here the anwer seems to be "no".

The previous lemma found properties of equilibria when there are nonzero equilibrium profits. We will now identify some properties of the payoffs and the sharing rule that must be satisfied in the first place to make non-zero profit equilibria possible. First we recall the following definition in [3]:

Definition 3 A function $f: \mathbb{R} \rightarrow \mathbb{R}$ is left lower semi-continuous (llsc) at $x \in \mathbb{R}$ if $\liminf _{x^{\prime} / x} f\left(x^{\prime}\right) \geq f(x)$. 
It is sufficient for $f$ being $l l s c$ if $f$ is lower semi-continuous or (leftcontinuous.

Lemma 2 Given a Bertrand game $\Gamma$, assume that $\pi^{\max }<\infty$ and that there exists a Nash equilibrium $\left(F_{1}^{*}, . ., F_{n}^{*}\right)$ with $J_{>} \neq \emptyset$.

1. Let $p \in \cup_{i \in J_{>}} P_{i}$. If $A^{*}(p, N)>1$ or $J_{=\neq} \neq \emptyset$ then either $\left\{\pi_{m}\right\}_{m \in M}$ is not tie-decreasing at $p$, or $\pi_{1}$ is not llsc at $p$.

2. If $J_{=\neq} \neq \emptyset$ then there are at most countably many prices $p<t$ with $\pi_{1}(p)>0$, and at these prices $\liminf _{p^{\prime} / p} \pi_{1}\left(p^{\prime}\right) \leq 0$.

This result outlines necessary conditions for Nash equilibria with positive profits for some firm. Excluding these conditions allows us to state our main result about the necessity of zero profits in any Bertrand equilibrium, be it pure or mixed, symmetric or asymmetric:

Theorem 1 Any Nash equilibrium of a Bertrand game $\Gamma$ involves zero expected profits for all players if all of the following hold:

1. maximum profits $\pi^{\max }$ are finite;

2. the sharing rule $\left\{\pi_{m}\right\}_{m \in M}$ is tie-decreasing given $\pi_{1}$;

3. profits $\pi_{1}$ are left lower semi-continuous at all $p \in \mathbb{R}_{+}$where $\pi_{1}(p)>0$.

Proof. If $\left|J_{>}\right|>1$ then by Lemma $1 A^{*}(t, N) \geq\left|J_{>}\right|>1$, and if $\left|J_{>}\right|=1$ then $J_{=\neq} \neq \emptyset$, both of which contradict assumptions 2 and 3 by Lemma 2 .

This theorem is a generalization of previous results on zero-payoff equilibria to arbitrary payoffs and sharing rules. The first assumption of Theorem 1 is needed to avoid "bootstrap equilibria" as in [2] and [8], where unlimited 
payoffs sustain positive equilibrium profits. The necessity of the second assumption is made clear by the result of [5] that with continuous payoffs and strictly increasing returns to scale, where equal sharing is not tie-decreasing everywhere, positive profit equilibria exist. Example 2 in [3] shows that also the third condition is needed to rule out positive equilibrium profits.

After clarifying the circumstances under which all equilibria must involve zero profits, we will now list some properties of payoffs and the sharing rule in zero-payoff Nash equilibria.

Lemma 3 Given a Bertrand game $\Gamma$, let $\left(F_{1}^{*}, . ., F_{n}^{*}\right)$ be a zero-payoff Nash equilibrium. Without loss of generality players are ordered such that $t_{1} \leq t_{2} \leq$ $\ldots \leq t_{n}$ where $t_{i}=\sup S_{i}, i \in N$. Let $s=\min \cup_{i \in N} S_{i}$. Then

1. $\pi_{1}(p) \leq 0$ for all $p \in[0, s)$ and for almost all $p \in\left[s, t_{2}\right]$. If $\pi_{1}(p)>$ 0 for $p \in\left[s, t_{2}\right]$ then $A^{*}(p, N)>1$ and there is $m \in M$ such that $\pi_{m}(p) \leq 0$.

2. For any $p \in \cup_{i \in N} P_{i}$ with $p<t_{1}$ either $\pi_{1}(p)=0$, or there is an $m \in M$ such that $\pi_{m}(p) \geq 0>\pi_{1}(p)$, or $\pi_{1}(p)>0$ and $\pi_{1}$ is not llsc at $p$.

By excluding the possibility of profitable deviations, this lemma places tight upper limits on the equilibrium supports of at least two players, and states that if $\pi_{1}$ is non-zero at equilibrium prices below $t_{1}$ then either the sharing rule is not tie-decreasing or that $\pi_{1}$ is not llsc. These results will be used in the following section to prove non-existence of any kind of Nash equilibrium in an example.

The second statement of this lemma is not a contradiction to Theorem 1 , but rather implies that when its assumptions apply then $\pi_{1}(p)=0$ for all $p \in \cup_{i \in N} P_{i}$ with $p<t_{1}$, and therefore $\pi_{1}(p) \leq 0$ for all $p<t_{1}$. 


\section{Sign-Preserving Sharing Rules}

In this section we highlight some of the special properties of Bertrand games with sign-preserving (SP) sharing rules. We start with an example where under equal sharing no Nash equilibrium exists, while the same game with winner-take-all sharing has a zero-payoff Nash equilibrium, as shown in [14, p. 118]. With equal sharing the sum of payoffs is not upper semi-continuous, violating one of the assumptions of the Dasgupta-Maskin existence theorem [4]. Neither is this game "better-reply secure" as defined by [10]: It is enough to consider the non-equilibrium point $p_{i}=\underline{p}_{1}$ for all $i \in N$. There are paths involving zero payoffs that converge to this point, but there is no deviation from it that results in strictly positive payoffs. The non-existence result is therefore not contradicted by these existence theorems.

Example: There are $n$ firms and demand is $D(p)=\max \{0,1-p\}$. Costs are $C(0)=0$ and $C(q)=F \in(0,1 / 4)$ for $q>0$. With equal sharing, we have $\pi_{1}(p)=(1-p) p-F>\pi_{m}(p)=(1-p) p / m-F$ whenever $\pi_{1}(p)>0$ and for any $m \in M$, thus equal sharing is tie-decreasing. Since $\pi_{1}$ is llsc where it is positive and maximum profits are finite, by theorem 1 there are no Nash equilibria involving positive profits for any firm.

$$
<<\text { HERE FIGURE } 2>>
$$

Assume that there is a zero-payoff equilibrium. The highest price $p^{*}$ such that there are only countably many $p \leq p^{*}$ with $\pi_{1}(p)>0$ is $p^{*}=\underline{p}_{1}$ (see Figure 2), thus from Lemma 3 it follows that $t_{2} \leq \underline{p}_{1}$. Yet $\pi_{1}\left(\underline{p}_{1}\right)=0$ and $\pi_{m}(p)<0$ for all $p<\underline{p}_{1}$ and all $m \in N$. Thus $s \geq \underline{p}_{1}$ and $\alpha_{1}^{*}\left(\underline{p}_{1}\right)=$ $\alpha_{2}^{*}\left(\underline{p}_{1}\right)=1$, which contradicts $\pi_{m}\left(\underline{p}_{1}\right)<0$ for all $m \in M$.

On the other hand, $p_{i}=\underline{p}_{1}$ for all $i \in N$ is a symmetric pure strategy equilibrium under winner-take-all sharing, where $\pi_{m}(p)=((1-p) p-F) / m$ for 
$p<1$. As we will see below, the equilibrium exists because this sharing rule is sign-preserving.

Equilibria under SP sharing have simple structure:

Lemma 4 Given a Bertrand game $\Gamma$ with SP sharing, in any Nash equilibrium either $J_{>}=N$ and $\pi_{1}(p)>0$ for all $p \in \cup_{i \in N} P_{i}$, or $J_{=}=N$ and $\pi_{1}(p)=0$ for all $p \in \cup_{i \in N} P_{i}$ with $p<t_{1}$.

This lemma implies that we need not worry about atoms, since under SP sharing atoms cannot hide payoffs of opposite sign.

We make the following definitions:

Definition 4 1. An "initial viable price" (IVP) is a price $s \in \mathbb{R}_{+}$such that $\pi_{1}(s) \geq 0$ and $\pi_{1}(p) \leq 0$ for all $p<s$. If also $\pi_{1}(s)=0$ then $s$ is an "initial break-even price" (IBP, notion introduced by [3]).

2. A function $f: \mathbb{R} \rightarrow \mathbb{R}$ is right upper semi-continuous (rusc) at $x \in \mathbb{R}$ if $\lim \sup _{x^{\prime} \backslash x} f\left(x^{\prime}\right) \leq f(x)$.

An IVP is unique unless it is an IBP, and both IVP's and IBP's are natural candidates for symmetric pure strategy equilibria. The following theorem provides sufficient conditions for their existence and clarifies their relation with Nash equilibria under SP sharing.

Theorem 2 Given a Bertrand game $\Gamma$ with SP sharing:

1. Assume that there is $\bar{p} \in \mathbb{R}_{+}$such that $\pi_{1}(\bar{p}) \geq 0$. An IVP exists if for all $p \in[0, \bar{p}]$ either $\pi_{1}$ is rusc or non-negative.

2. Any IVP $p \in \mathbb{R}_{+}$gives rise to a pure symmetric Nash equilibrium. 
3. Any IVP is an IBP if $\pi_{1}(0) \leq 0$ and $\pi_{1}$ is llsc.

4. Any IBP is unique if either $\pi_{1}$ is strictly quasi-concave, or if $\pi_{1}$ is strictly increasing in an open neighborhood around any IBP.

5. There is a zero-payoff Nash equilibrium if and only if there is an IBP.

If payoffs $\pi_{1}$ are not rusc then an IVP (and the corresponding Nash equilibrium) may fail to exist: Let $D(p)=1-p, C(q)=q / 2$ for $0 \leq q<1 / 2$, and $C(q)=q$ for $q \geq 1 / 2$, so that $\pi_{1}$ is right-discontinuous at $p=1 / 2$, with $\pi_{1}(1 / 2)<0$. By Theorem 1 and Lemma 3 no Nash equilibria exist. IVP's form symmetric pure Nash equilibria under SP sharing simply because undercutting does not pay off.

Without left-continuity of $\pi_{1}$ IBP's may not exist: See the example of Section 3, with $c(q)$ slightly above $1 / 4$ for $q<1 / 3$. On the other hand, statement 4 indicates when IBP's, and therefore symmetric pure strategy Nash equilibria with zero profits, are unique. This is the best uniqueness result that one can hope for, because if $n>2$ then zero-payoff equilibria are always non-unique because some player can choose actions above $t_{2}$ without upsetting the equilibrium. Furthermore, we must still invoke theorem 1 to rule out equilibria with positive payoffs.

Statement 5 is a generalization of Theorem 1 in [3] on the relations between IBP and zero-payoff equilibria. It depends decisively on the SP sharing assumption, which must be invoked for both the necessity and the sufficiency part ([3] invoke it for both without without stating it explicitly because they take the sharing rule as given). For arbitrary sharing rules the existence of an IBP is neither necessary nor sufficient for a zero-payoff equilibrium. The example at the beginning of this section shows the latter. The pure-strategy zero-payoff equilibrium $p_{i}=s, i \in N$, with $\pi_{n}(s)=0>\pi_{1}(s)$ in [5], under 
the equal sharing rule and decreasing returns to scale, proves the former.

This theorem brings us back to the classical Bertrand result of zero profits. There is a change in intuition, however, which is that the classical "competitive outcome" of price equal to marginal cost is the exception rather than the rule, while the generic outcome is price equal to average cost. These two coincide only under constant returns to scale.

Finally, we remark how continuity of the demand and cost functions in the Bertrand model translates into the continuity of payoffs:

Lemma 5 Let the demand function $D$ be non-increasing and the cost function $C$ be non-decreasing. If $D$ and $C$ are lower (upper) semi-continuous then $\pi_{1}$ is right- (left-) continuous.

Interestingly, it is lower and not upper semi-continuity of demand that guarantees the existence of equilibria. The reason is that demand discontinuities pose no problems concerning existence unless they are magnified through the cost function into upward jumps in profits. Right-continuity at these upward jumps then follows from $D$ being lower semi-continuous.

\section{$5 \quad$ Expectation sharing rules}

In this section we analyse a specific class of sharing rules in the Bertrand model, where $\pi_{1}(p)=p D(p)-C(D(q))$. These sharing rules are obtained by defining tied firms' payoffs as the expected value of profits over some random distribution of quantities. In principle, the total quantity attributed could be different from the total quantity demanded, but we will concentrate on the case where they coincide. Under this restriction the quantities of tied firms are not independent random variables, therefore we will consider joint 
distributions of quantities. Furthermore, since firms are symmetric we will only consider symmetric sharing rules.

First we need some definitions.

Definition 5 1. A joint distribution funtion $F_{p, m}$ on $[0, D(p)]^{m}$ is a symmetric sharing distribution if the order of its arguments is irrelevant. An expectation sharing rule is a family $F=\left\{F_{p, m}\right\}_{p \in \mathbb{R}_{+}, m \in M}$ of sharing distributions.

2. An expectation sharing rule is feasible if the supports of the joint probability distributions $F_{p, m}$ are contained in the sets

$$
\mathcal{F}_{m}(p)=\left\{\left(q_{1}, . ., q_{m}\right) \in[0, D(p)]^{m} \mid \sum_{i=1}^{m} q_{i}=D(p)\right\} .
$$

In this case $F_{p, m}$ is also called feasible. The sets of symmetric joint probability distributions on $\mathcal{F}_{m}(p)$ are $\Delta\left(\mathcal{F}_{m}(p)\right)$. The set of feasible and symmetric expectation sharing rules is $\mathcal{E}=\times_{p \in \mathbb{R}_{+}, m \in M} \Delta\left(\mathcal{F}_{m}(p)\right)$.

A sharing rule $\left\{\pi_{m}\right\}_{m \in M}$ is derived from $F \in \mathcal{E}$ by

$$
\pi_{m}(p)=E[p q-C(q) \mid p, m]=\int_{\mathcal{F}_{m}(p)}[p q-C(q)] d F_{p, m}\left(q, q_{2}, . ., q_{n}\right),
$$

for all $p \in \mathbb{R}_{+}$and $m \in M$. The set $\mathcal{E}$ is larger than the set of sharing rules $\left\{\pi_{m}\right\}_{m \in M}$, in the sense that for given $(D, C)$ there may be several expectation sharing rules $F \in \mathcal{E}$ that give rise to the same $\left\{\pi_{m}\right\}_{m \in M}$.

Expectation sharing rules have an interpretation in terms of demand composition. A simple rule defines feasible distributions $F_{p, m}$ for all pairs $(p, m)$ with:

- Equal sharing: The joint distributions $F_{p, m}$ give weight 1 to the center point $(D(p) / m, . ., D(p) / m)$, and the marginal distributions attribute 
weight 1 to $q=D(p) / m$. Its interpretation is that infinitely many small buyers distribute themselves independently.

- Winner-take-all sharing $W$ : The joint distributions $W_{p, m}$ give weight $1 / m$ to each of the $m$ corner points $(0, . ., 0, D(p), 0, . ., 0)$ of $\mathcal{F}_{m}(p)$, and the marginal distributions attribute weight $(m-1) / m$ at $q=0$ and $1 / m$ on $q=D(p)$. [3] interpret this sharing rule as representing a single large buyer who randomly turns to one of the firms.

Other distributions of quantities follow from mixtures of buyers of different size.

Feasibility is a reasonable but not necessary requirement on expectation sharing rules. ${ }^{1}$ Alternatively one can imagine sharing rules where some consumers "lose their way", so that total demand faced by firms is less than $D(p)$. Anyway, this notion has not appeared in the previous sections, so that the results obtained there do not depend on this assumption.

First we list some properties of expectation sharing rules:

Lemma 6 1. The set $\mathcal{E}$ of feasible and symmetric expectation sharing rules is convex.

2. If $F_{p, m} \in \Delta\left(\mathcal{F}_{m}(p)\right)$ for $(p, m) \in \mathbb{R}_{+} \times M$ then $E[q \mid p, m]=D(p) / m$ and

$$
\pi_{m}(p)=\frac{p D(p)}{m}-E[C(q) \mid p, m]
$$

Expectation sharing rules need not be tie-decreasing, as the example of equal sharing shows. The following lemma presents an exact characterization, and a sufficient condition on $C$.

\footnotetext{
${ }^{1}$ I would like to thank an anonymous referee for suggesting the discussion of feasibility.
} 
Lemma 7 Given $(D, C), F \in \mathcal{E}$ is tie-decreasing if and only if

$$
p D(p)<E\left[\sum_{i=1}^{m} C\left(q_{i}\right) \mid p, m\right]+m \pi_{1}(p)
$$

for all $m \in M$ and $p \in \mathbb{R}_{+}$such that $\pi_{1}(p)>0$. All $F \in \mathcal{E}$ are tie-decreasing if $C$ is concave with $C(0)=0$.

We will now determine when expectation sharing rules are sign-preserving. Given demand and cost functions $D$ and $C$, denote the sets of zero-atzero, or sign-preserving, feasible and symmetric expectation sharing rules as $\mathcal{E}^{z a z}(D, C)$ and $\mathcal{E}^{s p}(D, C)$, respectively. A generic element of these sets has the form $F=\left\{F_{p, m}\right\}_{p \in \mathbb{R}_{+}, m \in M}$. For any $(D, C)$

$$
\{W\} \subset \mathcal{E}^{s p}(D, C) \subset \mathcal{E}^{z a z}(D, C) \subset \mathcal{E} .
$$

In fact, we will consider an intermediate and two extreme versions of the above question:

1. For which class of demand and cost functions are all feasible and symmetric expectation sharing rules sign-preserving, i.e. $\mathcal{E}^{s p}(D, C)=\mathcal{E}$ ?

2. Which expectation sharing rules are sign-preserving for all demand and cost functions, i.e. which are the $F \in \mathcal{E}$ such that $F \in \mathcal{E}^{s p}(D, C)$ for all $(D, C)$ ? We already know that $W$ is such a sharing rule.

3. For which demand and cost functions do sign-preserving expectation sharing rules exist that are different from the ones identified in question 2 ? That is, which are the $(D, C)$ such that $\mathcal{E}^{s p}(D, C) \neq\{W\} ?$

The following Lemma provides the main tools for the analysis:

Lemma 8 1. Given demand and cost functions $D$ and $C, F \in \mathcal{E}^{s p}(D, C)$ if and only if, for all $p \in \mathbb{R}_{+}$and $m \in M$,

$$
p D(p) \gtreqless E\left[\sum_{i=1}^{m} C\left(q_{i}\right) \mid p, m\right] \text { if } \pi_{1}(p) \gtreqless 0 .
$$

The set $\mathcal{E}^{s p}(D, C)$ is convex. 
2. Given demand and cost functions $D$ and $C, F \in \mathcal{E}^{z a z}(D, C)$ if and only if for all prices $p_{0}$ such that $\pi_{1}\left(p_{0}\right)=0$ we have, for all $m \in M$,

$$
E\left[\sum_{i=1}^{m} C\left(q_{i}\right) \mid p_{0}, m\right]=C\left(q_{0}\right)
$$

where $q_{0}=D\left(p_{0}\right)$. The set $\mathcal{E}^{z a z}(D, C)$ is convex.

Conditions (8) and (10) demonstrate that the conditions of being tiedecreasing or sign-preserving are independent and do not contradict each other. More precisely, if a sharing rule is tie-decreasing and sign-preserving then

$$
E\left[\sum_{i=1}^{m} C\left(q_{i}\right) \mid p, m\right]<p D(p)<E\left[\sum_{i=1}^{m} C\left(q_{i}\right) \mid p, m\right]+m \pi_{1}
$$

for all $m \in M$ and $p \in \mathbb{R}_{+}$such that $\pi_{1}(p)>0$.

The convexity of the sets $\mathcal{E}^{z a z}(D, C)$ and $\mathcal{E}^{s p}(D, C)$ implies that either they contain only the winner-take-all sharing rule $W$ or they contain infinitely many elements.

Being zero-at-zero is a necessary condition for being sign-preserving. This is true even for profits $\pi_{1}$ which do not have an IBP; in this case the requirement of being zero-at-zero is automatically fulfilled. As for the first of the above two questions, considering zero-at-zero sharing rules already provides a partial answer. Let $P_{0}$ be the set of prices such that $\pi_{1}\left(p_{0}\right)=0$, and $P_{0}^{+}$ the set of prices $p_{0} \in P_{0}$ such that $D\left(p_{0}\right)>0$.

Lemma 9 Given any $(D, C)$,

1. $\mathcal{E}^{z a z}(D, C)=\mathcal{E}$ if and only if, for all $p_{0} \in P_{0}$ and $m \in M$,

$$
\sum_{i=1}^{m} C\left(q_{i}\right)=C\left(D\left(p_{0}\right)\right) \forall\left(q_{1}, . ., q_{m}\right) \in \mathcal{F}_{m}\left(p_{0}\right)
$$


2. if $P_{0}^{+}$is empty then $\mathcal{E}^{z a z}(D, C)=\mathcal{E}$;

3. if $P_{0}^{+}=\left\{p_{0}\right\}$ then

(a) if $n=2$, then $\mathcal{E}^{z a z}(D, C)=\mathcal{E}$ if and only if $C(q)=c q+f(q)$ on $\left[0, q_{0}\right]$, where $q_{0}=D\left(p_{0}\right), c=p_{0}$ and $f:\left[0, q_{0}\right] \rightarrow \mathbb{R}$ is an antisymmetric function around $q_{0} / 2$ with $f(0)=f\left(q_{0} / 2\right)=f\left(q_{0}\right)=$ 0

(b) if $n>2$, then $\mathcal{E}^{z a z}(D, C)=\mathcal{E}$ if and only if $C(q)=c q$ on $\left[0, D\left(p_{0}\right)\right]$, with $c=p_{0}$.

4. If $P_{0}^{+}$has more than one element, then $\mathcal{E}^{z a z}(D, C) \varsubsetneqq \mathcal{E}$.

The power of the first point of this Lemma is that it reduces the condition in Lemma 8 to a simple restriction on the cost function. Point 2 states that if $\pi_{1}$ has no zero then being zero-at-zero is a vacuous requirement on sharing rules. Point $3 \mathrm{a}$ is driven by the symmetry between the two players, while in point $3 \mathrm{~b}$ additional degrees of freedom rule out any deviations from linearity. The intuition of the fourth point is simple: Applying point 3 to two different prices $p_{0}$ and $p_{0}^{\prime}$ leads to a contradiction.

The previous lemma has also restricted the cases where $\mathcal{E}^{s p}(D, C)=\mathcal{E}$. Clearly $\mathcal{E}^{s p}(D, C) \varsubsetneqq \mathcal{E}$ if $P_{0}^{+}$has more than one element. If there are more than two firms, we have:

Corollary 3 Let $(D, C)$ be such that $P_{0}^{+}=\left\{p_{0}\right\}$ and $n>2$. If $\mathcal{E}^{s p}(D, C)=$ $\mathcal{E}$ then the cost function $C$ is linear on $\left[0, D\left(p_{0}\right)\right]$.

Clearly $\mathcal{E}^{s p}(D, L)=\mathcal{E}$ for any cost function $L$ that is linear on $[0, \infty)$, but the Corollary also allows for infinitely many cost functions that are non-linear above $D\left(p_{0}\right)$. 
We will now turn to the case $n=2$, and show that for most demand functions there exist non-linear cost functions such that $\mathcal{E}^{s p}(D, C)=\mathcal{E}$. The additional assumption that we must make is weak and can be relaxed if other functional forms are used in the construction.

Lemma 10 Let $n=2$ and $D$ be a non-increasing demand function. Assume that there is a price $p_{0}$ where $q_{0}=D\left(p_{0}\right)>0$ and $D$ is locally Lipschitzcontinuous: There are $\mu, \varepsilon>0$ such that $\left|D(p)-D\left(p_{0}\right)\right|<\mu\left|p-p_{0}\right|$ for all $p$ in an $\varepsilon$-neighborhood of $p_{0}$. Then there are infinitely many cost functions $C$ such that $\mathcal{E}^{s p}(D, C)=\mathcal{E}$, of the form $C(q)=c q+f(q)$, where $c=p_{0}$ and

$$
f(q)=\left\{\begin{array}{ccc}
A q\left(\frac{1}{2}-\frac{q}{q_{0}}\right)\left(1-\frac{q}{q_{0}}\right) & \text { if } & 0 \leq q \leq q_{0} \\
0 & \text { if } & q_{0}<q
\end{array},\right.
$$

for some $A \in(-2 \varepsilon, 0)$.

Now that we have answered the first question, the following lemma provides us with a fast answer to the second question: ${ }^{2}$

Lemma 11 The only expectation sharing rule that is sign-preserving for all demand and cost functions is the winner-take-all sharing rule: If $F \in \mathcal{E}$ and $F \neq W$ there are $(D, C)$ such that $F \notin \mathcal{E}^{s p}(D, C)$.

The proof shows that $W$ is the only sign-preserving expectation sharing rule if $C$ is strictly convex or concave.

To answer the third question, the following lemma describes a weak sufficient condition on demand and cost such that $W$ is not the only signpreserving sharing rule.

\footnotetext{
${ }^{2}$ We thank a referee for conjecturing this result.
} 
Lemma 12 Assume that for $(D, C)$ there is an IBP $p_{0}$ with $q_{0}=D\left(p_{0}\right)$ and for some $m_{0} \in M$ there are $q^{\prime}, q^{\prime \prime} \in \mathcal{F}_{m_{0}}\left(p_{0}\right)$ with $\sum_{i=1}^{m_{0}} C\left(q_{i}^{\prime}\right)>C\left(q_{0}\right)$ and $\sum_{i=1}^{m_{0}} C\left(q_{i}^{\prime \prime}\right)<C\left(q_{0}\right)$. Then there are infinitely many $F \in \mathcal{E}^{s p}(D, C)$ with $F \neq W$.

The condition on cost that drives this result can be interpreted as asking for sufficiently strong increasing returns to scale $\left(\sum_{i=1}^{m_{0}} C\left(q_{i}^{\prime}\right)>C\left(q_{0}\right)\right)$ and decreasing returns to scale $\left(\sum_{i=1}^{m_{0}} C\left(q_{i}^{\prime \prime}\right)<C\left(q_{0}\right)\right)$.

To sum up the preceding discussion: Winner-take-all sharing often is the only sign-preserving feasible and symmetric expectation sharing rule, but there is a large set of cases where either infinitely many or even all other such sharing rules are also sign-preserving.

\section{Conclusions}

We have analyzed Bertrand games from a different point of view. We did not take the distribution of payoffs at ties (the sharing rule) as given, but rather let it be the main object of analysis. To this effect we have determined which properties of Bertrand equilibria depend on the sharing rule, and under which circumstances only zero-profit equilibria exist. We have shown that for the class of sign-preserving sharing rules (zero-profit) equilibria exist under weak conditions, generalizing [3]. For other sharing rules the existence of equilibrium is not guaranteed. Finally, we have analised the class of expectation sharing rules, where payoff at ties is defined as expected profits over some random distribution of quantities.

On the conceptual level, the mode of analysis introduced in this paper may also be fruitful for the analysis of other games with discontinuous payoffs and equilibria involving ties with positive probability, for example auctions. 
Furthermore, we believe that the relation between our approach and endogenous sharing rules points to a fruitful avenue for further research.

\section{Appendix}

\section{Proof of Lemma 1:}

1. Fix $i \in N$, and let $p_{i}$ be any point in $S_{i}$, and $\phi_{i} \subset \mathbb{R}_{+}$any open neighborhood around this point. The set $\phi_{i} \cap S_{i}$ has positive mass under $F_{i}^{*}$, because otherwise $S_{i} \backslash \phi_{i}$ would be a strictly smaller closed set containing probability mass 1 , a contradiction to $S_{i}$ being the smallest such set by definition. This implies that $\phi_{i} \cap S_{i}$ contains members of $P_{i}$. Since this is true for all points $p_{i}$ and neighborhoods $\phi_{i}$, the set $P_{i}$ is dense in $S_{i}$ for all $i \in N$.

For $i \in N$, let $G_{i}^{*}(p)=1-F_{i}^{*}(p)+\alpha_{i}^{*}(p)=\operatorname{Pr}\left(p_{i} \geq p\right)$, which is leftcontinuous in $p$. Clearly, $\max \left\{\alpha_{i}^{*}(p), 1-F_{i}^{*}(p)\right\} \leq G_{i}^{*}(p) \leq 1$ for all $p \in$ $\mathbb{R}_{+}$, and $\lim _{p \rightarrow \infty} G_{i}^{*}(p)=0$. From (2), for all $i \in N$ and $p \in \mathbb{R}_{+}$,

$$
u\left(p, F_{-i}^{*}\right) \leq \sum_{T \subset N_{i}}\left|\pi_{|T|+1}(p)\right| \prod_{j \neq i} G_{j}^{*}(p) \leq 2^{n-1} \pi^{\max } \prod_{j \neq i} G_{j}^{*}(p)
$$

thus $\lim _{p \rightarrow \infty} u\left(p, F_{-i}^{*}\right)=0$. For all $i \in J_{>}$the support $S_{i}$ must therefore have a finite maximum $t_{i}$, and $\sup P_{i}=t_{i}$ because $P_{i}$ is dense in $S_{i}$. Let $t=\min _{i \in J_{>}} t_{i}$. If there is any $j \in J_{>}$with $t_{j}>t$, we have $u_{j}\left(p, F_{-j}^{*}\right)=0$ for all $p \in\left(t, t_{j}\right]$ since $G_{i}^{*}(p)=0$ for at least one firm $i$ with $t_{i}=t$. This is a contradiction to $\sup P_{j}=t_{j}$, thus $t_{j}=t$ for all $j \in J_{>}$.

By (14), for every $i \in N$

$$
\limsup _{p \nearrow t} u\left(p, F_{-i}^{*}\right) \leq 2^{n-1} \pi^{\max } \prod_{j \neq i} \lim _{p \nearrow t} G_{j}^{*}(p)=2^{n-1} \pi^{\max } \prod_{j \neq i} G_{j}^{*}(t) .
$$

If there is any $j \in N_{i}$ such that $G_{j}^{*}(t)=0$ this implies that $\lim \sup _{p / t} u\left(p, F_{-i}^{*}\right) \leq$ 0 , which is a contradiction to $\Pi_{i}>0$ and $\sup P_{i}=t$ if $i \in J_{>}$. Therefore 
$G_{j}^{*}(t)>0$ for all $j \in N_{i}$ if $i \in J_{>}$, which implies $F_{j}^{*}(t)<1+\alpha_{j}^{*}(t)$ for all $j \in J_{=}$, and $t \in P_{j}$ and $\alpha_{j}^{*}(t)>0$ for all $j \in J_{>} \backslash\{i\}$ if $\left|J_{>}\right|>1$. The statement for $J_{>}=\{i\}$ follows from the proof in the next paragraph applied to $t=\sup P_{i}$. Note that in this case we cannot conclude that $t \in P_{i}$.

For any two firms $j, k \in N, j \neq k$, we can write firm $j$ 's expected profits at price $p$ as

$$
u\left(p, F_{-j}^{*}\right)=\alpha_{k}^{*}(p) U_{j k}(p, 2)+\left(1-F_{k}^{*}(p)\right) U_{j k}(p, 1)
$$

where, with $N_{j k}=N \backslash\{j, k\}$,

$$
U_{j k}(p, a)=\sum_{T \subset N_{j k}} \pi_{|T|+a}(p) \prod_{i \in T} \alpha_{i}^{*}(p) \times \prod_{l \in N_{j k} \backslash T}\left(1-F_{l}^{*}(p)\right) .
$$

Since at $p=t$ we have $1-F_{j}^{*}(t)=0$ if $j \in J_{>}$, it follows that $u\left(t, F_{-k}^{*}\right)=$ $\alpha_{j}^{*}(t) U_{j k}(t, 2)$ for any $k \in N_{i}$.

If $k \in J_{>}$then $u\left(t, F_{-k}^{*}\right)=\Pi_{k}$ and $u\left(t, F_{-j}^{*}\right)=\alpha_{k}^{*}(t) U_{k j}(t, 2)=\Pi_{j}$, from which follows $\alpha_{k}^{*}(t) \Pi_{k}=\alpha_{j}^{*}(t) \Pi_{j}$ since $U_{j k}(p, a)=U_{k j}(p, a)$. Since the pairing $(j, k)$ was arbitrary, $\alpha_{i}^{*}(t) \Pi_{i}=K$ for all $i \in J_{>}$and some $K>0$.

If on the other hand $k \in J_{=}$and $\alpha_{j}^{*}(t)>0$, which is definitely true if $\left|J_{>}\right|>1$, then necessarily $U_{j k}(t, 2) \leq 0$. Since $\alpha_{k}^{*}(t)=0$ if $U_{j k}(t, 2)<0$, in any case $\alpha_{k}^{*}(t) U_{j k}(t, 2)=0$, and $u\left(t, F_{-j}^{*}\right)=\left(1-F_{k}^{*}(t)\right) U_{j k}(t, 1)=\Pi_{j}>0$, from which follows that $1-F_{k}^{*}(t)>0$.

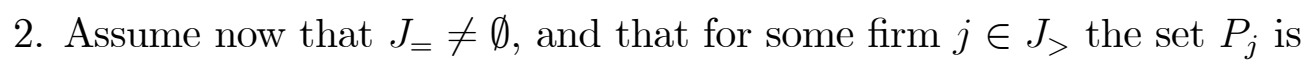
uncountable. Since all $S_{i}, i \in N$, can contain only countably many atoms, $P_{j}$ then contains a price $\hat{p}<t$ such that $\alpha_{j}^{*}(\hat{p})=\alpha_{k}^{*}(\hat{p})=0$ for some firm $k \in J_{=}$. Payoffs at this price are

$$
\begin{aligned}
& u_{j} \equiv u\left(\hat{p}, F_{-j}^{*}\right)=\left(1-F_{k}^{*}(\hat{p})\right) U_{j k}(\hat{p}), \\
& u_{k} \equiv u\left(\hat{p}, F_{-k}^{*}\right)=\left(1-F_{j}^{*}(\hat{p})\right) U_{j k}(\hat{p}) .
\end{aligned}
$$


Since $F_{j}^{*}(\hat{p})<1, u_{j}=\Pi_{j}>0$ and $u_{k} \leq 0$ contradict each other. The sets $P_{j}$, $j \in J_{>}$, are therefore countable, and can contain only atoms and a countable number of limit points of atoms. These atoms contain all probability mass. The sets $S_{j}, j \in J_{>}$, do therefore not contain any open sets without atoms.

It also follows from the above argument that any price $p<t$ in $P_{j}$, for some firm $j \in J_{>}$, and which is not an atom for firm $j$, must be an atom for all firms $i \in J_{=}$.

Finally, any price $p<t$ which is not an atom for any firm gives rise to positive profits if $\pi_{1}(p)>0$. Therefore in an equilibrium with $J_{=} \neq \emptyset$ this price must either be an atom of some firm in $J_{>}$, or of at least two firms in $J_{=}$.

\section{Proof of Lemma 2:}

1. For each $p \in P_{i}$ for some $i \in J_{>}$it follows from $u\left(p, F_{-i}^{*}\right)>0$ that $\prod_{j \neq i} G_{j}^{*}(p)>0$. Letting

$$
R_{i l}^{*}(p)=\sum_{\substack{T \subset N_{i} \\|T|=l-1}} \prod_{j \in T} \frac{\alpha_{j}^{*}(p)}{G_{j}^{*}(p)} \times \prod_{k \in N_{i} \backslash T}\left(1-\frac{\alpha_{k}^{*}(p)}{G_{k}^{*}(p)}\right)
$$

for all $l \in N$ and $p \in P_{i}$, we can write payoffs (2) as

$$
u\left(p, F_{-i}^{*}\right)=\prod_{j \neq i} G_{j}^{*}(p) \times \sum_{l=1}^{n} R_{i l}^{*}(p) \pi_{l}(p) .
$$

The term $R_{i l}^{*}(p)$ is the probability that $(l-1)$ firms are tied with firm $i$ at price $p$, given that all firms $j \in N_{i}$ choose prices greater or equal than $p$. Then $0 \leq R_{i l}^{*}(p) \leq 1$ and $\sum_{l=1}^{n} R_{i l}^{*}(p)=1$. Therefore, the expression $\sum_{l=1}^{n} R_{i l}^{*}(p) \pi_{l}(p)$ is a weighted average of $\left\{\pi_{1}(p), . ., \pi_{n}(p)\right\}$, and is strictly positive since $p \in P_{i}$.

As in $\cup_{j \neq i} S_{j}$ there are only countably many atoms there is an increasing sequence $\left\{p_{l}\right\}_{l=1}^{\infty} \subset \mathbb{R}_{+}$, with $\lim _{l \rightarrow \infty} p_{l}=p$ and $A^{*}\left(p_{l}, N_{i}\right)=0$ for all $l \in \mathbb{N}$. 
By underbidding $p$ slightly, firm $i$ can guarantee itself, in the limit, at least the payoff

$$
\begin{aligned}
\liminf _{l \rightarrow \infty} \pi_{1}\left(p_{l}\right) \prod_{j \neq i} G_{j}\left(p_{l}\right) & =\prod_{j \neq i} G_{j}(p) \liminf _{l \rightarrow \infty} \pi_{1}\left(p_{l}\right) \\
& \geq \prod_{j \neq i} G_{j}(p) \liminf _{p^{\prime} / p} \pi_{1}\left(p^{\prime}\right) .
\end{aligned}
$$

Since $p \in P_{i}$ it is necessary that $\sum_{l=1}^{n} R_{i l}^{*}(p) \pi_{l}(p) \geq \liminf _{p^{\prime} / p} \pi_{1}\left(p^{\prime}\right)$. If $\pi_{1}$ is llsc at $p$ if follows that $\sum_{l=1}^{n} R_{i l}^{*}(p) \pi_{l}(p) \geq \pi_{1}(p)$.

Now if $A^{*}(p, N) \geq 2$ then there is $j \in N_{i}$ such that $\alpha_{j}^{*}(p)>0$, and thus $R_{i 1}^{*}(p)<1$. It follows that there is an $m \in M$ such that $\pi_{m}(p)>0$ and $\pi_{m}(p) \geq \pi_{1}(p)$.

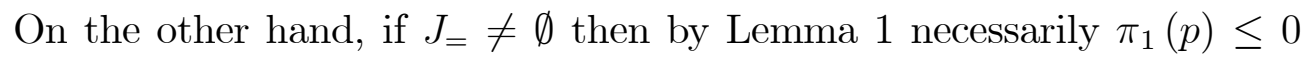
for almost all prices $p<t$ since there are only countably many atoms in $\cup_{i \in N} S_{i}$. Therefore $\liminf _{p^{\prime} / \bar{p}} \pi_{1}\left(p^{\prime}\right) \leq 0$ for all $\bar{p}<t$. If $\pi_{1}$ is llsc at $p \in P_{i}$ then $\pi_{1}(p) \leq 0$, from which follows that there is an $m \in M$ such that $\pi_{m}(p)>0 \geq \pi_{1}(p)$ since $\sum_{l=1}^{n} R_{i l}^{*}(p) \pi_{l}(p)>0$.

\section{Proof of Lemma 3:}

First note that $s=\min \cup_{i \in N} S_{i}$ exists since all $S_{i}$ are closed and bounded from below.

1. It is clear that $\pi_{1}(p) \leq 0$ for all $p<s$ because otherwise a deviation to a $p$ with $\pi_{1}(p)>0$ would lead to positive payoffs. Since there are only countably atoms in $\cup_{i \in N} S_{i}$, almost all $p<t_{2}$ yield player $1 u\left(p, F_{-1}^{*}\right)=$ $\pi_{1}(p) \prod_{j \in N_{1}}\left(1-F_{j}^{*}(p)\right)$, which is positive unless $\pi_{1}(p) \leq 0$. On the other hand, if $\pi_{1}(p)>0$ then $A^{*}\left(p, N_{1}\right)>0$, and there is $m \in M$ such that $\pi_{m}(p) \leq 0$

2. For $i \in N$ let $p \in P_{i}$ with $p<t_{1}$. Then $G_{j}^{*}(p)>0$ for all $j \in N_{i}$, and $\sum_{k=1}^{n} R_{i k}^{*}(p) \pi_{k}(p)=0$, and there is $m \in M$ with $\pi_{m}(p) \geq 0$ if $\pi_{1}(p)<0$. Otherwise either $\pi_{1}(p)=0$, or $\pi_{1}(p)>0$ with $\liminf _{p^{\prime} / p} \pi_{1}\left(p^{\prime}\right) \leq 0$. 


\section{Proof of Lemma 4:}

Let $i \in J_{>} \neq \emptyset$ and $j \in J_{=} \neq \emptyset$. Since $u\left(p, F_{-i}^{*}\right)>0$ for $p \in P_{i}$, SP sharing implies that $\pi_{m}(p)>0, m \in N$. Thus $u\left(p, F_{-j}^{*}\right)>0$, a contradiction. If $J_{=}=N$ then $u\left(p, F_{-i}^{*}\right)=0$ for $p \in P_{i}$ implies $\pi_{m}(p)=0$ for all $m \in N$ if $p<t_{1}$.

\section{Proof of Theorem 2:}

1. Define $p^{*} \leq \bar{p}$ as $p^{*}=\inf \left\{0 \leq p \leq \bar{p} \mid \pi_{1}(p) \geq 0\right\}$. Either $\pi_{1}\left(p^{*}\right) \geq 0$, or by upper semi-continuity from the right we must have $0 \leq \lim \sup _{p \backslash p^{*}}$ $\pi_{1}(p) \leq \pi_{1}\left(p^{*}\right)$. By definition of $p^{*}, \pi_{1}(p)<0$ for all $p<p^{*}$, thus $p^{*}$ is an IVP.

2. If $p$ is an IVP, by SP sharing $\pi_{n}(p) \geq 0$. Playing $p$ with probability 1 is a pure symmetric equilibrium since uncercutting does not increase payoffs.

3. Let $p_{0}>0$ be an IVP. Since $\pi_{1}$ is llsc at $p_{0}$ :

$$
0 \geq \lim _{p \nearrow p_{0}} \inf \pi_{1}(p) \geq \pi_{1}\left(p_{0}\right) \geq 0,
$$

i.e. $\pi_{1}\left(p_{0}\right)=0$. If $p_{0}=0$ is an IVP then $\pi_{1}(0) \geq 0$ by definition. By assumption $\pi_{1}(0) \leq 0$, so again $\pi_{1}\left(p_{0}\right)=0$.

4. Let $p_{l}<p_{h}$ be IBP's. If $\pi_{1}$ is strictly quasi-concave then $\pi_{1}(p)>0$ for all $p \in\left(p_{l}, p_{h}\right)$. If $\pi_{1}$ is strictly increasing in an open neighborhood around $p_{l}$, then there are an $\varepsilon \in\left(0, p_{h}-p_{l}\right)$ and $p \in\left(p_{l}, p_{l}+\varepsilon\right)$ with $\pi_{1}(p)>0$. Both contradict $p_{h}$ being an IBP.

5. An IBP $p$ is an IVP with $\pi_{1}(p)=0$ and leads to a zero-payoff Nash equilibrium by point 2. For the converse, assume there is a zero-payoff Nash equilibrium with firms ordered such that $t_{1} \leq . . \leq t_{n}$. For any $p \in P_{1}$, $u\left(p, F_{-1}^{*}\right)=0$ and by SP sharing $\pi_{1}(p)=0$. By Lemma $3, \pi_{1}(r) \leq 0$ for all $r \in[0, s)$. Any $p \in\left[0, t_{1}\right]$ with $\pi_{1}(p)>0$ would lead to $u\left(p, F_{-1}^{*}\right)>0$ under SP sharing, thus $\pi_{1}(p) \leq 0$ for all $p \in\left[0, t_{1}\right]$. Since $P_{1} \subset\left[s, t_{1}\right]$ is non-empty the result follows. 


\section{Proof of Lemma 5:}

Let $D$ and $C$ be lower semi-continuous. Since $D$ is non-increasing and $C$ is non-decreasing, $D$ is right- and $C$ is left-continuous. Therefore $\pi_{1}$ is right-continuous. The analogous result holds if $D$ and $C$ are upper semicontinuous.

\section{Proof of Lemma 6:}

Given any $(p, m) \in \mathbb{R}_{+} \times M$, if $F_{p, m}, G_{p, m} \in \Delta\left(\mathcal{F}_{m}(p)\right)$ then for any $\lambda \in[0,1]$ the function $\lambda F_{p, m}+(1-\lambda) G_{p, m}$ is also a symmetric and feasible sharing distribution on $\mathcal{F}_{m}(p)$. As for the second statement, by symmetry and feasibility we obtain, for any $(p, m)$ and $i \in\{1, . ., m\}$, $m E[q \mid p, m]=\sum_{i=1}^{m} E\left[q_{i} \mid p, m\right]=E\left[\sum_{i=1}^{m} q_{i} \mid p, m\right]=E[D(p) \mid p, m]=D(p)$.

\section{Proof of Lemma 7:}

If $\left(q_{1}, . ., q_{m}\right) \in \mathcal{F}_{m}(p)$ then by symmetry $m E[C(q) \mid p, m]=E\left[\sum_{i=1}^{m} C\left(q_{i}\right) \mid p, m\right]$, so

$$
\pi_{m}=\frac{1}{m}\left(p D(p)-E\left[\sum_{i=1}^{m} C\left(q_{i}\right) \mid p, m\right]\right)
$$

The statement then follows from $\pi_{1}(p)>\pi_{m}(p)$ whenever $\pi_{1}(p)>0$. Rearranging terms differently leads to

$$
\pi_{1}(p)>\frac{1}{m-1}\left(C(D(p))-E\left[\sum_{i=1}^{m} C\left(q_{i}\right) \mid p, m\right]\right) .
$$

If $C$ is concave with $C(0)=0$ then $C(q) / q \leq C(\lambda q) / \lambda q$ for any $q>0$ and $\lambda \in(0,1)$. For $0<q_{1} \leq q_{2}$ it follows that

$$
C\left(q_{1}+q_{2}\right) \leq\left(q_{1}+q_{2}\right) \frac{C\left(q_{2}\right)}{q_{2}} \leq q_{1} \frac{C\left(q_{1}\right)}{q_{1}}+q_{2} \frac{C\left(q_{2}\right)}{q_{2}}=C\left(q_{1}\right)+C\left(q_{2}\right),
$$


which also holds if $q_{1}=0$. By induction, $C(D(p))=C\left(\sum_{i=1}^{m} q_{i}\right) \leq$ $\sum_{i=1}^{m} C\left(q_{i}\right)$ for any $\left(q_{1}, . ., q_{m}\right) \in \mathcal{F}_{m}(p)$. This makes the right-hand side of (16) non-positive.

\section{Proof of Lemma 8:}

By definition, the sharing rule $F=\left\{F_{p, m}\right\}_{p \in \mathbb{R}_{+}, m \in M} \in \mathcal{E}$ is sign-preserving if, for all $p \in \mathbb{R}_{+}$and $m \in M, E[p q-C(q) \mid p, m] \gtreqless 0$ if $\pi_{1}(p) \gtreqless 0$. By Lemma 6 , and summing over $i=1, . ., m$ we obtain the result. Let $\left\{F_{p, m}\right\},\left\{G_{p, m}\right\} \in$ $\mathcal{E}^{s p}$. Then for any $\lambda \in[0,1]$ and all $p \in \mathbb{R}_{+}$and $m \in M$,

$$
E_{\lambda F_{p, m}+(1-\lambda) G_{p, m}[.]}=\lambda E_{F_{p, m}}[.]+(1-\lambda) E_{G_{p, m}}[.] .
$$

As concerns the second statement, it follows immediately from the first statement and $\pi_{1}\left(p_{0}\right)=p_{0} D\left(p_{0}\right)-C\left(q_{0}\right)=0$.

\section{Proof of Lemma 9:}

1. If $\sum_{i=1}^{m} C\left(q_{i}\right)=C\left(q_{0}\right) \forall q \in \mathcal{F}_{m}\left(p_{0}\right)$ for all $p_{0} \in P_{0}$ and $m \in M$, then for any $F \in \mathcal{E}$

$$
E\left[\sum_{i=1}^{m} C\left(q_{i}\right) \mid p_{0}, m\right]=E\left[C\left(q_{0}\right) \mid p_{0}, m\right]=C\left(q_{0}\right)
$$

and by Lemma $8 F \in \mathcal{E}^{z a z}(D, C)$. For the reverse, consider some $p_{0} \in P_{0}$ and some $m^{\prime} \in M$ such that there is a $q^{\prime} \in \mathcal{F}_{m^{\prime}}\left(p_{0}\right)$ with $\sum_{i=1}^{m^{\prime}} C\left(q_{i}^{\prime}\right)=K \neq$ $C\left(q_{0}\right)$. Let $F_{p_{0}, m^{\prime}}$ be the sharing distribution that gives equal weight to all permutations of $q^{\prime}$ and zero weight to the rest of $\mathcal{F}_{m^{\prime}}\left(p_{0}\right)$. Then clearly

$$
E\left[\sum_{i=1}^{m^{\prime}} C\left(q_{i}\right) \mid p_{0}, m^{\prime}\right]=K \neq C\left(q_{0}\right),
$$

and the resulting expectation sharing rule is not zero-preserving.

2. This point is obvious since being zero-at-zero implies no restriction in this case. 
3a. Assume that $C(q)=c q+f(q)$ as described in the statement. Then for any $\left(q_{1}, q_{2}\right) \in \mathcal{F}_{2}\left(p_{0}\right)$ we have $q_{2}=q_{0}-q_{1}$ and

$$
\begin{aligned}
C\left(q_{1}\right)+C\left(q_{0}-q_{1}\right) & =c q_{0}+f\left(q_{1}\right)+f\left(q_{0}-q_{1}\right) \\
& =c q_{0}=C\left(q_{0}\right) .
\end{aligned}
$$

For the converse, let $c=C\left(q_{0}\right) / q_{0}$ and $f(q)=C(q)-c q$ on $\left[0, q_{0}\right]$, which implies $f\left(q_{0}\right)=0$. For any point $\left(q_{1}, q_{0}-q_{1}\right) \in \mathcal{F}_{2}\left(p_{0}\right)$ it follows that

$$
C\left(q_{1}\right)+C\left(q_{0}-q_{1}\right)=c q_{0}+f\left(q_{1}\right)+f\left(q_{0}-q_{1}\right) .
$$

Condition 13 can only be fulfilled if $f\left(q_{1}\right)+f\left(q_{0}-q_{1}\right)=0$. If this is to be true for all $q_{1} \in\left[0, q_{0}\right]$ it implies that $f$ is anti-symmetric around $q_{0} / 2$ and that $f(0)=f\left(q_{0} / 2\right)=0$. This proves statement $3 \mathrm{a}$.

3b. Clearly $\mathcal{E}^{z a z}(D, C)=\mathcal{E}$ if $C(q)=c q$ on $\left[0, D\left(p_{0}\right)\right]$ because $\sum_{i=1}^{m} C\left(q_{i}\right)=$ $c \sum_{i=1}^{m} q_{i}=c q_{0}$. Now for the converse. Given the result of point $3 \mathrm{a}$ we will prove that $f(q)=0$ on $\left[0, q_{0}\right]$ once we allow $m>2$. Consider $m=3$. Then for any point $\left(q_{1}, q_{2}, q_{3}\right) \in \mathcal{F}_{3}\left(p_{0}\right)$,

$$
\sum_{i=1}^{3} C\left(q_{i}\right)=c q_{0}+\sum_{i=1}^{3} f\left(q_{i}\right) .
$$

Condition 13 then implies that $\sum_{i=1}^{3} f\left(q_{i}\right)=0$ for any $\left(q_{1}, q_{2}, q_{3}\right) \in \mathcal{F}_{3}\left(p_{0}\right)$. Letting $\delta \in\left[0, q_{3}\right]$ we arrive at two more identities:

$$
\begin{aligned}
& f\left(q_{1}+\delta\right)+f\left(q_{2}\right)+f\left(q_{3}-\delta\right)=0 \\
& f\left(q_{1}\right)+f\left(q_{2}+\delta\right)+f\left(q_{3}-\delta\right)=0 .
\end{aligned}
$$

Taking differences leads to

$$
f\left(q_{1}+\delta\right)-f\left(q_{1}\right)=f\left(q_{2}+\delta\right)-f\left(q_{2}\right)
$$

Since $q_{1}, q_{2}$ and $\delta$ are arbitrary this means that the function $f$ must have a constant slope, which given $f(0)=f\left(q_{0}\right)=0$ is only possible if $f$ is identically zero. 
4. Let $p_{1}, p_{2} \in P_{0}^{+}$with $p_{1}<p_{2}$ and note that point $3 \mathrm{a}$ of this Lemma applies to both $p_{1}$ and $p_{2}$. Let $m=2$ and assume that $C(q)=c q+f(q)$ on $\left[0, D\left(p_{1}\right)\right]$ with $f\left(D\left(p_{1}\right)\right)=f\left(D\left(p_{2}\right)\right)=0$. Then $\pi_{1}\left(p_{i}\right)=\left(p_{i}-c\right) D\left(p_{i}\right)=$ 0 implies $p_{1}=p_{2}=c$, a contradiction.

\section{Proof of Lemma 10:}

Choose $\varepsilon$ such that $\varepsilon \leq\left(\frac{1}{2}-\frac{1}{6} \sqrt{3}\right) \frac{q_{0}}{\mu}$. We will proceed in four steps.

1. Identify a sufficient condition on $f$ which is independent of the sharing rule.

Fix an expectation sharing rule $F \in \mathcal{E}$. For $F \in \mathcal{E}^{s p}(D, C)$ we must have

$$
(p-c) D(p) \gtreqless E[f(q)+f(D(p)-q) \mid p, 2] \forall p \gtreqless p_{0} .
$$

Let

$$
\bar{g}(q)=\max _{0 \leq z \leq q}\{f(z)+f(q-z)\}, \underline{g}(q)=\min _{0 \leq z \leq q}\{f(z)+f(q-z)\},
$$

then

$$
\underline{g}(D(p)) \leq E[f(q)+f(D(p)-q) \mid p, 2] \leq \bar{g}(D(p))
$$

because the distributions $F_{p, 2}$ are symmetric. Therefore it is sufficient for $F \in \mathcal{E}^{s p}(D, C)$ if both

$$
\begin{aligned}
& (p-c) D(p)>\bar{g}(D(p)) \forall p>p_{0}, \\
& (p-c) D(p)<\underline{g}(D(p)) \forall p<p_{0} .
\end{aligned}
$$

2. Compute the functions $\bar{g}$ and $\underline{g}$.

If $q<q_{0}$ then the maximum of $f(z)+f(q-z)$ is attained at $z=0$, with

$$
\bar{g}(q)=f(q)=A q\left(\frac{1}{2}-\frac{q}{q_{0}}\right)\left(1-\frac{q}{q_{0}}\right) .
$$


For $q>q_{0}$, the minimum of $f(z)+f(q-z)$ is attained at $z=q-q_{0}$, with

$$
\underline{g}(q)=\left\{\begin{array}{ccc}
\underline{g}_{1}(q)=-3\left(1-\frac{q}{q_{0}}\right)\left(1-\frac{2}{3} \frac{q}{q_{0}}\right)\left(1-\frac{q}{2 q_{0}}\right) A q_{0} & \text { if } & q \leq\left(\frac{3}{2}-\frac{1}{6} \sqrt{3}\right) q_{0} \\
\underline{g}_{2}(q)=\frac{1}{12 \sqrt{3}} A q_{0} & \text { if } & q>\left(\frac{3}{2}-\frac{1}{6} \sqrt{3}\right) q_{0}
\end{array} .\right.
$$

3. Show that $(p-c) D(p)>\bar{g}(D(p)) \forall p>p_{0}$

We have $D(p) \geq D\left(p_{0}\right)-\mu\left(p-p_{0}\right)>0$ (because $\varepsilon<\frac{q_{0}}{\mu}$ ) for $p \in$ $\left(p_{0}, p_{0}+\varepsilon\right)$. Let $l(q)=p_{0}+\varepsilon-\frac{\varepsilon q}{q_{0}}$ with $l\left(q_{0}\right)=p_{0}$ and $l(0)=p_{0}+\varepsilon$. Then $(p-c) D(p) \geq(l(D(p))-c) D(p)$, and

$$
(l(q)-c) q=\varepsilon\left(1-\frac{q}{q_{0}}\right) q>\bar{g}(q)=A q\left(\frac{1}{2}-\frac{q}{q_{0}}\right)\left(1-\frac{q}{q_{0}}\right)
$$

if $1>\frac{A}{\varepsilon}\left(\frac{1}{2}-\frac{q}{q_{0}}\right)$. Sufficient for this is $1>-\frac{1}{2} \frac{A}{\varepsilon}$ or $A>-2 \varepsilon$.

4. Show that $(p-c) D(p)<\underline{g}(D(p)) \forall p<p_{0}$.

Since $A>-2 \varepsilon$, we have $A>-2 \varepsilon(3+\sqrt{3}) \geq-2 \frac{q_{0}}{\mu}$ since $\varepsilon \leq\left(\frac{1}{2}-\frac{1}{6} \sqrt{3}\right) \frac{q_{0}}{\mu}$. Then, for $p \in\left(p_{0}-\varepsilon, p_{0}\right)$,

$$
\begin{aligned}
D(p) & <D\left(p_{0}\right)-\mu\left(p-p_{0}\right)<D\left(p_{0}\right)+2 \frac{q_{0}}{A}\left(p-p_{0}\right), \\
(p-c) D(p) & <(p-c) q_{0}<-\frac{A}{2}\left(1-\frac{D(p)}{q_{0}}\right) q_{0} \leq \underline{g}_{1}(q) .
\end{aligned}
$$

We must check that indeed $q=D\left(p_{0}-\varepsilon\right) \leq\left(\frac{3}{2}-\frac{1}{6} \sqrt{3}\right) q_{0}$, which is true since

$$
D\left(p_{0}-\varepsilon\right) \leq D\left(p_{0}\right)+\mu \varepsilon \leq\left(\frac{3}{2}-\frac{1}{6} \sqrt{3}\right) q_{0} .
$$

Now for $p<p_{0}-\varepsilon$

$$
(p-c) D(p)<\left(p_{0}-\varepsilon-c\right) D\left(p_{0}-\varepsilon\right) \leq-\varepsilon q_{0}<\underline{g}_{2}(q)
$$

if $A>-12 \sqrt{3} \varepsilon$ which is weaker than $A>-2 \varepsilon$.

Proof of Lemma 11: 
Consider demand and cost functions $D$ and $C$ which give rise to an IBP $p_{0}$. Furthermore, assume that $C$ is strictly convex with $C(0)=0$. For any $\left(q_{1}, . ., q_{m}\right) \in \mathcal{F}_{m}\left(p_{0}\right)$, we have $\sum_{i=1}^{m} q_{i}=q_{0}$. On the other hand, since $C$ is strictly convex with $C(0)=0$ then $\sum_{i=1}^{m} C\left(q_{i}\right)<C\left(q_{0}\right)$ unless there is some $j \in\{1, . ., m\}$ such that $q_{j}=q_{0}$ and $q_{i}=0$ for all $i \neq j$. In this case $\sum_{i=1}^{m} C\left(q_{i}\right)=C\left(q_{0}\right)$ holds. That is, equality is only obtained at the $m$ corner points of $\mathcal{F}_{m}\left(p_{0}\right)$, therefore by Lemma 8 all probability mass must be concentrated there. By symmetry, each corner point has a mass of $1 / m$, which is the probability distribution of the winner-take-all sharing rule. Therefore only $W$ is in $\mathcal{E}^{z a z}(D, C)$, and by implication in $\mathcal{E}^{s p}(D, C)$.

\section{Proof of Lemma 12:}

For simplicity, we will construct a set of sharing rules in $E^{s p}(D, C)$ different from $W$ only at $\left(p_{0}, m_{0}\right)$. Let $F_{p, m}$ be equal to the winner-take-all sharing distribution for all $(p, m) \in \mathbb{R}_{+} \times M \backslash\left\{\left(p_{0}, m_{0}\right)\right\}$. Let $F^{\prime}, F^{\prime \prime} \in \Delta\left(\mathcal{F}_{m_{0}}\left(p_{0}\right)\right)$ be the two sharing distributions that attribute equal weight to all permutations of the tuples $q^{\prime}$ and $q^{\prime \prime}$, respectively. Then for $F^{\prime}$,

$$
E_{F^{\prime}}\left[\sum_{i=1}^{m_{0}} C\left(q_{i}\right) \mid p_{0}, m_{0}\right]=\sum_{i=1}^{m_{0}} C\left(q_{i}^{\prime}\right)
$$

with the corresponding result for $F^{\prime \prime}$. Let

$$
\lambda=\frac{C\left(q_{0}\right)-\sum_{i=1}^{m_{0}} C\left(q_{i}^{\prime \prime}\right)}{\sum_{i=1}^{m_{0}} C\left(q_{i}^{\prime}\right)-\sum_{i=1}^{m_{0}} C\left(q_{i}^{\prime \prime}\right)} \in(0,1),
$$

and define $F_{p_{0}, m_{0}}=\lambda F^{\prime}+(1-\lambda) F^{\prime \prime}$, which is also a member of $\Delta\left(\mathcal{F}_{m_{0}}\left(p_{0}\right)\right)$ because it is a convex set. Then

$$
\begin{aligned}
& E_{F_{p_{0}, m_{0}}}\left[\sum_{i=1}^{m_{0}} C\left(q_{i}\right) \mid p_{0}, m_{0}\right]=\lambda E_{F^{\prime}}\left[\sum_{i=1}^{m_{0}} C\left(q_{i}\right) \mid p_{0}, m_{0}\right] \\
& +(1-\lambda) E_{F^{\prime \prime}}\left[\sum_{i=1}^{m_{0}} C\left(q_{i}\right) \mid p_{0}, m_{0}\right] \\
& =\lambda \sum_{i=1}^{m_{0}} C\left(q_{i}^{\prime}\right)+(1-\lambda) \sum_{i=1}^{m_{0}} C\left(q_{i}^{\prime \prime}\right) \\
& =C\left(q_{0}\right) \text {, }
\end{aligned}
$$


which implies that $F_{p_{0}, m_{0}}$ is zero-at-zero and $F \in \mathcal{E}^{s p}(D, C) \backslash\{W\}$. Since $\mathcal{E}^{s p}(D, C)$ is convex, any convex combination between $F$ and $W$ is also a member of $\mathcal{E}^{s p}(D, C)$. 


\section{References}

[1] Balder, E. J.: On Equilibria for Discontinuous Games: Continuous Nash Approximation Schemes. mimeo, Mathematical Institute, University of Utrecht (March 2002)

[2] Baye, M. R., Morgan, J.: A Folk Theorem for One-Shot Bertrand Games. Economics Letters 65, 59-65 (1999)

[3] Baye, M. R., Morgan, J.: Winner-take-all Price Competition. Economic Theory 19, 271-282 (2002)

[4] Dasgupta, P., Maskin, E.: The Existence of Equilibrium in Discontinuous Economic Games, I:Theory. Review of Economic Studies 53, 1-26 (1986)

[5] Dastidar, K. G.: On the Existence of Pure Strategy Bertrand Equilibrium. Economic Theory 5(1), 19-32 (1995)

[6] Harrington, J. E.: A Re-Evaluation of Perfect Competition as the Solution to the Bertrand Price Game. Mathematical Social Sciences 17(3), $315-328$ (1989)

[7] Hoernig, S. H.: Mixed Bertrand Equilibria under Decreasing Returns to Scale: An Embarrassment of Riches. Economics Letters 74(3), 359-62 (2002)

[8] Kaplan, T.R., Wettstein, D.: The Possibility of Mixed-Strategy Equilibria with Constant-Returns-to-Scale Technology under Bertrand Competition. Spanish Economic Review 2(1), 65-71 (2000)

[9] Osborne, M. J., Pitchik, C.: Price Competition in a CapacityConstrained Duopoly. Journal of Economic Theory 38, 238-260 (1986) 
[10] Reny, P. J.: On the Existence of Pure and Mixed Strategy Nash Equilibria in Discontinuous Games. Econometrica 67(5), 1029-56 (1999)

[11] Sharkey, W. W., Sibley, D. S.: A Bertrand Model of Pricing and Entry. Economics Letters 41(2), 199-206 (1993)

[12] Simon, L. K., Zame, W. R.: Discontinuous Games and Endogenous Sharing Rules. Econometrica 58(4), 861-72 (1990)

[13] Sion, M., Wolfe, P.: On a Game Without a Value, Annals of Mathematics Studies 39, 299-306 (1957)

[14] Vives, X.: Oligopoly Pricing: Old Ideas and New Tools. Cambridge, MA: MIT Press 1999 
Figure Legends:

Figure 1: One firm may make positive equilibrium profits.

Figure 2: No pure or mixed equilibria because of increasing returns to scale and equal sharing. 


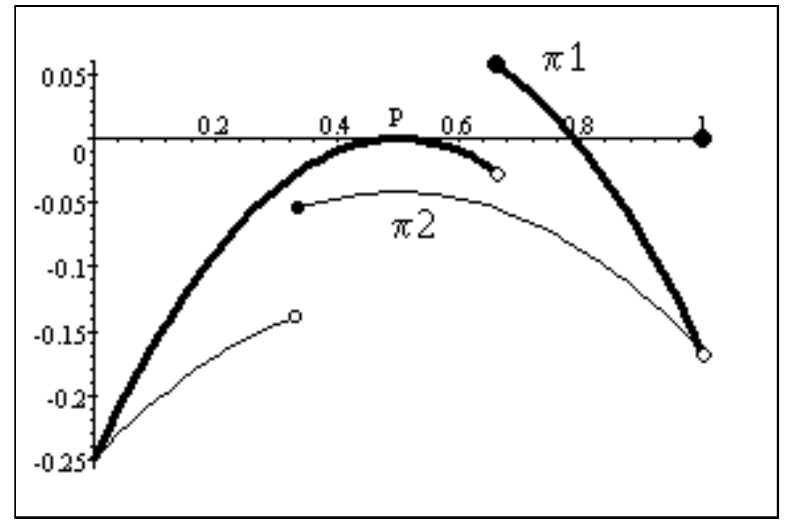

Figure 1: The Nash Equilibria may involve both positive and zero profits. 


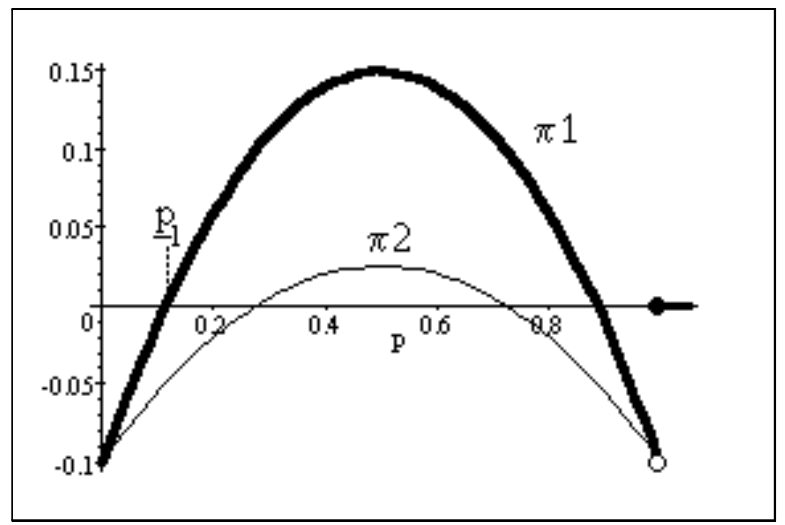

Figure 2: No pure nor mixed Nash equilibria exist. 